\title{
Where the Wild Things Are: Pathogenesis of SIV Infection in African Nonhuman Primate Hosts
}

\author{
Ivona Pandrea • Cristian Apetrei
}

Published online: 27 January 2010

(C) The Author(s) 2010. This article is published with open access at Springerlink.com

\begin{abstract}
African nonhuman primates that are natural hosts of simian immunodeficiency virus (SIV) are generally spared from disease progression. Pathogenic and nonpathogenic SIV infections share some major features: high viral replication, massive acute depletion of mucosal $\mathrm{CD}^{+}{ }^{+} \mathrm{T}$ cells, and partial control of the virus by both adaptive and innate immune responses. A key distinction of natural SIV infections is rapid and active control of immune activation and apoptosis of $\mathrm{T}$ cells that contributes to the integrity of mucosal barrier and lack of microbial translocation. This allows partial recovery of $\mathrm{CD}^{+} \mathrm{T}$ cells and preservation of the function of other immune cell subsets. A better understanding of the mechanisms underlying the lack of disease in natural hosts for SIV infection will likely provide important clues as to the therapy of HIV-1 infection.
\end{abstract}

Keywords Simian immunodeficiency virus · Virus replication $\cdot \mathrm{CD} 4+\mathrm{T}$ cells $\cdot$ Immune activation $\cdot$ Innate immunity $\cdot$ African green monkey $\cdot$ Sooty mangabey

\section{Introduction}

Without treatment, HIV-1 infection progresses to AIDS in 7 to 10 years. The survival of HIV-infected patients consid-

I. Pandrea $(\bowtie)$

Center for Vaccine Research and Department of Pathology,

University of Pittsburgh,

9014 Biomedical Science Tower 3, 3501 Fifth Avenue,

Pittsburgh, PA 15261-9045, USA

e-mail: pandrea@cvr.pitt.edu

C. Apetrei

Center for Vaccine Research and Department of Microbiology and Molecular Genetics, School of Medicine,

University of Pittsburgh,

Pittsburgh, PA, USA erably increased with the use of highly active antiretroviral therapy (HAART). Nowadays, a newly HIV-infected 20year-old who receives appropriate treatment early in the infection has a life expectancy of at least 69 years. Although these results are encouraging and unprecedented, the adverse effects, prohibitive costs, and the issues of viral resistance to antiretroviral drugs are still limiting factors of HAART. Complementary approaches to antiretroviral therapy, aimed at upholding immune system function through immunotherapies, are likely needed to transform HIV infection into a chronic, life-lasting, treatable condition.

This new focus on the development of immunotherapies as an approach to prevent HIV disease progression is supported by numerous lines of evidence that have accumulated during recent years and generated a new paradigm in which excessive immune activation and T-cell proliferation are major determinants of progression to AIDS $[1,2]$. Particularly, this new concept is fueled by data from the study of persistent nonprogressive simian immunodeficiency virus (SIV) infection in natural African nonhuman primate (NHP) hosts [2].

In this review, we address the major aspects of SIV pathogenesis in natural hosts, with emphasis on the mechanisms of the lack of disease progression.

\section{Simian Immunodeficiency Viruses}

More than 40 SIVs naturally infect wild African NHPs, with high prevalence [3]. In spite of being designated SIV, by analogy with HIV, these viruses generally do not induce immune suppression in their hosts [2]. This fundamental difference is equally fascinating and intriguing. Obviously, there is an important question raised by the lack of disease progression of SIV infection in natural hosts: are these highly divergent, apparently harmless viruses that infect 
distant monkey species instructive for HIV infection? Rapidly accumulating data on the pathogenesis of SIV infection in natural hosts and in new hosts upon crossspecies transmission indicate that these "exotic" models may actually hold clues regarding the immune correlates of the control of SIV/HIV disease progression [2].

First, the viruses infecting African NHPs retained their pathogenic potential. This is illustrated by their ability to induce immunosuppression and disease progression in new hosts upon cross-species transmission [3]. HIV-1 and HIV2 , the two viruses causing AIDS in humans, resulted from cross-species transmissions from chimpanzees/gorillas and sooty mangabeys (SMs), respectively [3]. Also, accidental transmission of SIVsmm, which naturally infects SMs, to different species of Asian macaques resulted in progressive infections in the new hosts [4]. Moreover, rare AIDS cases were reported in several African NHP species infected with their species-specific or cross-species transmitted SIVs [5]. These cases were described during long-term follow-up after extensive incubation periods in captive animals that outlived the normal species lifespan in the wild. They recapitulate all virologic, immunologic, and clinical conditions that usually define AIDS in humans [5].

Second, the benign coexistence of SIVs and their African NHP hosts resulted from their interactions over millennia [3], and a long-term adaptation of the primate hosts to prevent the deleterious consequences of lentiviral infection. Although the mechanisms through which the nonpathogenicity was achieved are still under debate, this adaptation resulted in a pattern of chronic, persistent, lifelasting infection with an incubation period that normally exceeds the lifespan of the species [5]. Because this is the target of current therapeutic approaches in HIV infection, the study of SIV infection in natural hosts can provide invaluable clues with regard to which aspects of HIV pathogenesis have to be manipulated to successfully control disease progression.

\section{Particularities of the Immune System of Natural Hosts of SIV}

The long-term virus-host coevolution left immunologic imprints in the natural hosts of SIVs.

The CCR5 expression (protein and mRNA) by $\mathrm{CD}^{+} \mathrm{T}$ cells from periphery, lymphoid, and mucosal tissues was found to be significantly lower in uninfected natural hosts, such as African green monkeys (AGMs), mandrills, SMs, solatus monkeys, and patas monkeys, compared with progressive hosts, such as rhesus macaques (RMs), pigtailed macaques, and humans [6]. Interestingly, the $\mathrm{CD}^{+} \mathrm{T}$ cells isolated from natural hosts express normal levels of CCR5, thus indicating that the finding is not a technical artifact [6]. Furthermore, CCR5 expression on the $\mathrm{CD} 4^{+} \mathrm{T}$ cells was particularly low in newborn AGMs and mandrills, compared with adults of the same species. Differently from RMs, in which CCR5 levels rapidly increase in infants of 6 months of age, reaching levels similar to those expressed by adults, such increases could not be observed in infant and juvenile African monkeys less than 3 years old [7•].

The red-capped mangabey (RCM) is an extreme example of a natural host that totally lacks CCR5 expression, as a result of a high prevalence $(83 \%)$ in this species of a delta-24a deletion [8] that prevents CCR5 gene transcription. This situation recalls the minority of humans who have a delta-32 deletion in the CCR5 gene structure that renders them resistant to HIV infection or disease progression [9]. Differently from HIV-1, which mainly uses CCR5 as a coreceptor, SIVrcm (the virus that naturally infects RCMs without clinical consequences) adapted to use a CCR2 or CCR4 coreceptor to ensure persistence in the RCM host [10].

These observations led to our original hypothesis that the changes in CCR5 expression in natural hosts reflect the coevolution of the viruses with their hosts [6]. This hypothesis is supported by the observation that, in baboons, CCR5 expression on $\mathrm{CD}^{+} \mathrm{T}$ cells is similar to that observed in humans and macaques [6]. Baboons are not natural hosts for SIV and progress to AIDS upon SIV/HIV2 infections [5].

In chimpanzees, CCR 5 expression on $\mathrm{CD} 4{ }^{+} \mathrm{T}$ cells is at intermediate levels between humans and natural NHP hosts [6], in line with molecular analyses that showed that chimpanzees acquired SIVcpz through cross-species transmissions from other monkey species, thus emerging more recently than the SIVs infecting African monkey species [3]. Moreover, SIVcpz is pathogenic in chimpanzees [11•].

To conclude, CCR5 expression by $\mathrm{CD}^{+} \mathrm{T}$ cells correlates with the ability of NHP species to develop or resist disease progression when infected with HIV/SIV.

The second difference in the immune system of the natural versus progressive hosts is that some African NHP species, such as AGMs, patas, and solatus monkeys, have low numbers of $\mathrm{CD}^{+} \mathrm{T}$ cells [12]. These low levels of $\mathrm{CD}^{+} \mathrm{T}$ cells appear to be characteristic only for African guenons (tribe Cercopithecini), as SMs and mandrills (tribe Papionini) were reported to have higher $\mathrm{CD}^{+} \mathrm{T}$ cell counts, similar to RMs, which belong to the same tribe.

Over the years, it was also reported that a large proportion of the $\mathrm{CD}^{+} \mathrm{T}$ cells do not express either CD4 or CD8, in natural hosts $[13,14 \cdot, 15,16]$. Recently, these double-negative cells where shown to arise through CD4 downregulation, as the cells enter the memory pool. This downregulation is independent of SIV infection. The $\mathrm{CD} 4^{\text {neg }}$ memory $\mathrm{T}$ cells maintain functions attributed to $\mathrm{CD}^{+} \mathrm{T}$ cells, including production of interleukin (IL)-2 
and IL-17, histocompatibility complex class II restriction, and expression of CD40 ligand and of forkhead box P3 [13, 14•]. In contrast with the double-negative $T$ cells observed in HIV-infected patients (which also occur through downregulation of CD4 [17]), the double-negative cells from AGMs are not SIV infected [14•]. Preservation of a cell subset that maintains $\mathrm{CD}^{+}$T-cell function while being resistant to SIV infection in vivo may be responsible for the lack of disease progression in natural hosts. The precise impact of the double-negative T-cell population for SIV transmission and pathogenesis remains to be determined.

Recent studies reported differences in the functionality of dendritic cells between pathogenic and nonpathogenic hosts. Thus, two studies reported that AGM and SM plasmacytoid dendritic cells (pDCs) produce markedly less interferon (IFN)- $\alpha$ in response to SIV and other Toll-like receptor (TLR) 7 and 9 ligands ex vivo [18, 19]. Such an observation may suggest that, in contrast with progressive hosts in which chronic stimulation of pDCs by SIV/HIV may drive the unrelenting immune system activation and dysfunction underlying AIDS progression, such a vicious cycle of continuous virus replication and immunopathology is absent in natural hosts.

These particularities of the immune system of natural hosts may have significant impact on the pathogenicity of SIV infection in these species (Table 1). One can imagine that the low levels of target cells (cells expressing CD4 and CCR5) may result in lower rates of SIV transmission or lower viral replication in natural hosts than in new hosts. Also, the low expression of CCR5 on the $\mathrm{CD} 4^{+} \mathrm{T}$ cells and a reduced capacity of the innate immunity to activate the adaptive immune system may result in lower levels of immune activation in the natural host than in progressive infections.

\section{SIV Mucosal Transmission in Natural Hosts}

The observation that natural hosts of SIV have low levels of $\mathrm{CCR} 5^{+} \mathrm{CD} 4^{+} \mathrm{T}$ cells at mucosal sites raised the question of whether the virus can be transmitted through mucosal routes in these species. This hypothesis was also suggested by the observation that in offspring and juvenile monkeys, which have even lower levels of $\mathrm{CCR}^{+} \mathrm{CD}^{+}$cells at mucosal sites, breastfeeding transmission is significantly lower than in pathogenic infection [7•]. However, the prevalence of SIV infection in wild populations of natural hosts significantly increases in adult animals, suggesting sexual transmission [3].

Experiments in mandrills failed to demonstrate any breastfeeding transmission of SIVmnd-1, even when mandrill dames were infected on the day of the delivery [7•]. In spite of very high viral load (VL) levels during the acute and chronic infection of the dames, and of high levels of replicating virus in milk during acute infection, no case of SIVmnd-1 transmission to offspring was observed during the lactation period. The design of this study, with dames being infected after delivery, precluded protection of the offspring through passively transmitted maternal antibodies. The lack of breastfeeding transmission in natural hosts was therefore correlated with the low CCR5 expression at mucosal sites [7•].

In a recent experiment, we assessed for the first time the efficacy of mucosal SIV transmission in a natural host by performing a dose-escalation study, in which AGMs and pigtailed macaques were intrarectally exposed to SIVagm (Pandrea, Unpublished data). This experiment demonstrated that natural hosts of SIVs can be infected by mucosal transmission, but at higher doses compared with pigtailed macaques. The efficacy of transmission was lower in juvenile animals than in adult AGMs, also suggesting that transmissibility depends on the availability of target cells at the mucosal sites. All these findings point toward a strong correlation between the levels of CCR5 expression on the $\mathrm{CD}^{+} \mathrm{T}$ cells and the rates of SIV transmission. Our studies also suggest that blockage of CCR5 may be an efficient treatment in preventing breastfeeding transmission, in addition to vaginal or rectal transmission.

\section{SIV Replication in Natural Hosts}

Despite the low levels of target cells, SIV replicates to high levels in natural hosts [20]. During acute infection, VLs are in the same range as those reported for pathogenic HIV/SIV infections [21]. During chronic infection of natural hosts, the levels of viral replication are slightly higher [20] than those observed in HIV-1-infected patients [22]. In contrast with pathogenic infections, the levels of viral replication are remarkably stable for decades during chronic infection of natural hosts $[23,24]$.

There are several observations that have to be stressed with regard to SIV replication in the natural host. First, similar to humans and macaques, plasma viremia during chronic infection is predictive of disease progression. The levels of chronic viral replication were significantly higher in African monkeys that progressed to AIDS than in those that resisted progression [5]. Note, however, that even these progressor monkeys showed a remarkable resilience to high levels of viral replication, which were maintained for decades prior to disease progression [5]. Second, SIV VLs in natural hosts appear to be dependent on the availability of $\mathrm{CD}^{+}{ }^{+} \mathrm{T}$ cells. Multiple lines of evidence support this conclusion: 1) In AGMs, a lower baseline level of $\mathrm{CD} 4^{+} \mathrm{T}$ cells associates steady-state levels of viral replication that are 1 log lower than in SMs and mandrills [20]; 2) In SMs 
Table 1 Comparison between pathogenic and nonpathogenic SIV infection

\begin{tabular}{|c|c|c|}
\hline Parameter & Persistent progressive infection & Persistent nonprogressive infection \\
\hline Acute viral replication & High & High \\
\hline Chronic viral replication & $\begin{array}{l}\text { High and short length set point followed } \\
\text { loss of control }\end{array}$ & High but indefinitely maintained set point \\
\hline Mutation rates & High & High \\
\hline Tissue reservoirs & Lymph nodes, intestine, brain & Lymph nodes, intestine, brain \\
\hline Target cells & Mainly lymphocytes, secondary macrophages & Mainly lymphocytes, secondary macrophages \\
\hline Peripheral CD4+ T cells & $\begin{array}{l}\text { Massive acute depletion; continuous loss } \\
\text { during chronic infection }\end{array}$ & $\begin{array}{l}\text { Massive acute depletion; almost } \\
\text { complete recovery during chronic phase }\end{array}$ \\
\hline Mucosal CD4+ T cells & $\begin{array}{l}\text { Massive acute depletion; continuous loss } \\
\text { during chronic infection }\end{array}$ & $\begin{array}{l}\text { Massive acute depletion; partial recovery } \\
\text { during the chronic phase }\end{array}$ \\
\hline Tregs & Depleted during the chronic infection & Preserved during the chronic infection \\
\hline $\mathrm{T}$ helper 17 cells & Depleted during the chronic infection & Preserved during the chronic infection \\
\hline T-cell proliferation & Accelerated & Normal \\
\hline T-cell activation & Increased & Normal \\
\hline Proinflammatory cytokines & Increased & Normal \\
\hline Anti-inflammatory cytokines & Delayed increase during acute infection & Very early increase during acute infection \\
\hline Apoptosis & Increased & Normal \\
\hline Functionality of immune cells & Impaired & Normal \\
\hline Cellular immune responses & Moderate & Moderate \\
\hline Humoral immune responses & Moderate & Moderate \\
\hline Intestinal barrier & Damaged & Maintained \\
\hline Microbial translocation & Increased & Absent \\
\hline CCR5 expression on CD4+ T cells & High & Very low \\
\hline
\end{tabular}

SIV simian immunodeficiency virus

and AGMs with severe $\mathrm{CD}^{+}$T-cell depletion after SIV infection, VLs decreased significantly [25]; 3) Experimental induction of immune activation in chronically infected AGMs resulted in a dramatic increase in the number of $\mathrm{CD}^{+} \mathrm{T}$ cells and VLs [26.]; and 4) As expected from previous studies in AGMs, experimental $\mathrm{CD}^{+}$T-cell depletion in SMs resulted in a significant decrease of VLs [27].

The low CCR5 expression observed in uninfected African NHPs does not appear to have a major impact on virus replication in the natural host. This paradox may be explained through several hypotheses. First, CCR5 expression may increase in response to SIV infection. The CCR5 ${ }^{+}$ $\mathrm{CD}^{+} \mathrm{T}$ cells are targeted and depleted by the SIV. Therefore, SIV-associated changes in the $\mathrm{CD} 4^{+} \mathrm{CCR} 5^{+}$ T-cell pool may reflect the opposing forces of both SIV replication (which reduces this cellular pool) and immune activation (which increases it). In AGMs, CCR5 expression by $\mathrm{CD} 4^{+} \mathrm{T}$ cells (both protein and mRNA levels) increases during acute SIVagm infection, as well as at certain time points during chronic infection. These increases in the CCR5 expression on the $\mathrm{CD} 4^{+} \mathrm{T}$ cells may be sufficient to expand the pool of SIV target cells and maintain the high VLs. Being only transient, these increases in CCR5 expression are not harmful to the hosts by not triggering excessive immune activation. Second, natural hosts may keep the CCR5 expression under control through an active mechanism, which remains to be determined. Finally, the lack of correlation between coreceptor expression and the levels of viral replication may be due to SIVs having other target cells in natural hosts.

Indeed, the paradox of SIV infection in natural hosts defined by association of a paucity of $\mathrm{CD} 4^{+} \mathrm{CCR} 5^{+}$target cells with high VLs also raised the question of whether in natural hosts SIVs could target cells other than $\mathrm{CD}^{+} \mathrm{T}$ lymphocytes. This hypothesis is also prompted by the observation that peripheral $\mathrm{CD}^{+}{ }^{+} \mathrm{T}$ cells are well preserved in natural hosts during chronic infection, almost at preinfection levels. Such alternative potential target cell candidates may be either the macrophages or $\mathrm{CD} 4^{+} \mathrm{T}$-cell subsets that do not express CCR5.

In some AGMs, the levels of SIVagm in the brain are as high as in humans with HIV-dementia [28], and the SIVagm strains isolated from the brain of infected AGMs are macrophage tropic. In the same animals, the SIVagm strains isolated from the lymph nodes were more restricted to growth in human T-cell lines [28]. These results suggest compartmentalization in the central nervous system without 
associated neuropathology and provide additional evidence that the nonpathogenic nature of SIV infection in natural hosts is not due to differences in the tissue and cell tropism from those for HIV-1-infected patients or SIV-infected macaques [28].

Similar to pathogenic infections, the bulk of viral replication is supported in natural hosts by lymphocytes rather than macrophages during both acute and chronic infection:

1) A dramatic depletion of $\mathrm{CD} 4^{+} \mathrm{T}$ cells occurs during acute infection in natural hosts $[29,30]$. At mucosal sites, depletion is of the same magnitude as in progressive infections $[29,30]$ and strongly suggests that nonpathogenicity of SIV infection in natural hosts is not due to preservation of mucosal $\mathrm{CD} 4^{+} \mathrm{T}$ cells. Both naïve and memory $\mathrm{CD} 4^{+} \mathrm{T}$-cell subsets are massively depleted at mucosal sites during acute SIV infection $[29,30]$. T helper (Th) 17 cells were recently reported to be preserved in natural hosts $[13,31]$. With the postacute partial control of viral replication and immune activation, a partial restoration of mucosal $\mathrm{CD}^{+} \mathrm{T}$ cells occurs during chronic infection in natural hosts [29, 30]. Altogether, these results demonstrate that the magnitude of acute mucosal $\mathrm{CD}^{+}$T-cell depletion cannot predict progression to AIDS [29] and that prolonged survival correlates with the levels of $\mathrm{CD} 4^{+} \mathrm{T}$-cell recovery.

2) During the acute infection, in situ studies demonstrated that SIVs mainly colocalize with $\mathrm{T}$ cells, colocalization with macrophages being negligible.

3) Viral dynamics studies in natural hosts also showed that viral replication occurs in short-lived cells that have a shorter average lifespan than those reported for HIV-infected humans and SIVmac-infected macaques $[32,33]$.

On the other hand, SIVagm.sab and other viruses infecting natural hosts were reported to be dual tropic in vitro and to use both CCR5 and CXCR4 [34], being therefore conceivable that the main target cells of these viruses are other $\mathrm{CD}^{+}$ T-cell subpopulations (ie, those expressing CXCR4). However, in vivo, SIVagm severely depletes the $\mathrm{CD}^{+} \mathrm{T}$ cells from the intestine (which more frequently express CCR5) and not from the blood or lymph nodes (that have a higher CXCR4 expression) in both nonprogressive (AGMs) [29] and cross-species transmitted (RMs and pigtailed macaques) infections [29]. This observation suggests that the virus is in fact CCR5 tropic in vivo.

Altogether these data show that SIVs naturally infecting African NHPs have the same biology and similar target cells compared with the lentiviruses that infect humans or macaques.

\section{Immune Responses to SIV Infection in Natural Hosts}

Studies suggested that SIV infection in natural hosts is independent of immune responses. The main argument is that, differently from elite controllers in which the lack of disease progression is achieved through control of viral replication [35], such control is not observed in natural hosts [20]. Note, however, that the stability of VLs over decades strongly supports an active (albeit partial) control of viral replication in natural hosts.

Numerous studies determined that, in natural hosts, the levels of anti-SIV binding and neutralizing antibodies are either low or in the same range as those reported for HIV-1 infection [36]. Natural hosts usually lack anti-Gag antibodies $[36,37]$. The significance of this observation is still debated, as the Gag antigens appear to be expressed, and the Gag-specific T-cell responses are comparable to those observed in pathogenic infections [37]. Neutralizing antibodies of variable potency can be found in the majority of SIV-seropositive AGMs [38, 39], and their detection is dependent on the virus strain and the cells used for the assay [38]. Most SIVsmm strains are resistant to neutralization [40]. These observations suggest that the low levels of antibodies or the weak neutralization activity may be responsible for the high viremia in these species [36]. Given the contradictory results of different studies, the potential role of humoral immune responses for the control of viral replication in natural hosts was assessed in AGMs by ablating anti-SIV antibody production through a longterm depletion of B cells. This study demonstrated that humoral immune responses do not play a determinant role in controlling viral replication in the natural host [39]. This is also true for SIVsmm-infected macaques that did not show significant changes in the levels of viral replication or disease progression when depleted of B cells and infected with a neutralization-sensitive SIVsmm strain [40].

In contrast, cellular immune responses observed in natural hosts seem to be involved in the control of viral replication [37, 41, 42], with a direct correlation between the levels of cellular immune responses and plasma VLs being reported in SMs [43]. However, cellular immune responses do not appear to be superior to those observed during SIV infection of RMs [43].

Recently, several groups performed experimental in vivo CD8 cell depletion studies in natural hosts and reported that administration of anti-CD8 monoclonal antibodies during both acute or chronic SIV infection resulted in increases in the levels of virus replication in both AGMs [44] and SMs [45]. During acute infection, CD8 cell depletion did not result in a significant increase of peak viremia, but in a plateau of high VLs after the peak, which were controlled with the recovery of $\mathrm{CD} 8^{+} \mathrm{T}$ 
cells. During chronic SIV infection, CD8 cell depletion in vivo also resulted in an increase of viral replication in natural hosts. Dual $\mathrm{CD} 8^{+}$and $\mathrm{CD} 20^{+}$cell depletion studies also pointed to cellular immune responses as the main factors involved in the control of viral replication in natural hosts [44]. None of the in vivo immune cell depletion studies reported any case of disease progression. This is in line with the above observation that even in the face of higher levels of viral replication, progression to AIDS occurs only after very long incubation periods in natural hosts [5].

\section{Low Immune Activation is Pathognomonic for SIV Infection in Natural Hosts}

The assessment of the various contributing factors that enable natural hosts of SIV to resist progression to AIDS thus revealed that the lack of disease progression in natural hosts is not due to 1) lower levels of SIV replication; 2) preservation of peripheral or mucosal $\mathrm{CD}^{+} \mathrm{T}$ cells during acute infection; 3) replication in cell populations that are different in natural versus pathogenic SIV infections; or 4) exquisite SIV-specific cellular or humoral immune responses.

The main difference identified between natural SIV infections and pathogenic HIV/SIV infections relied on the normal levels of immune activation and inflammation observed in natural hosts. Generalized immune activation is an important driver of viral replication disease progression in pathogenic HIV/SIV infections [1]. Chronic SIV infection in natural hosts is associated with 1) low levels of $\mathrm{CD}^{+}$and $\mathrm{CD}^{+}$T-cell activation and proliferation in the periphery, as well as in lymphoid and mucosal tissues [29, 46]; 2) no significant increased levels of $\mathrm{CD}^{+}$T-cell apoptosis in vivo or in vitro [29, 47]; 3) absence of cellcycle dysregulation associated with pathogenic HIV/SIV infections [48]; 4) no significant increase in the levels of proinflammatory cytokines compared with uninfected monkeys [26•, 39]; and 5) preserved function of the T-cell regenerative compartment. During acute SIV infection of natural hosts, the levels of T-cell activation and proliferation, although transiently increased, are blunted compared with those observed during pathogenic SIVmac infection of RMs, with a lower peak and a more rapid and pronounced postpeak decline of immune activation [29].

The low-level immune activation in natural hosts does not appear to represent ignorance of, or tolerance to, SIV antigens because SIV-specific $\mathrm{T}$ cells are present during natural SIV infections and exert immune pressure on the virus [41]. Moreover, an anti-inflammatory milieu was reported to be rapidly established in AGMs after SIVagm infection, characterized by an early increase in numbers of Tregs, paralleled by an increase in transforming growth factor- $\beta$ and IL-10 levels in the plasma very early after infection [49]. This anti-inflammatory milieu may actively attenuate the immune activation during natural SIV infections. These observations conflict with a recent study in SMs that reported that natural hosts intrinsically lack the ability to respond to TLR stimulation in vitro [18]. However, in contrast with the in vitro data showing the incapacity of pDCs to produce IFN- $\alpha$, numerous recent in vivo studies reported a transient increase of IFN- $\alpha$ production during acute infection in AGMs and SMs and an increased capacity of pDCs to produce IFN- $\alpha$ after infection. These increases, however, are lower than those observed in SIVmac-infected RMs and of very short duration, with IFN- $\alpha$ production returning to baseline levels by the time viral replication is controlled.
Fig. 1 Several mechanisms are involved in the control of immune activation in natural hosts of simian immunodeficiency viruses. In turn, normal levels of immune activation may result in prevention of disease progression through several pathways. $p D C s$ plasmacytoid dendritic cells; TLR Toll-like receptor

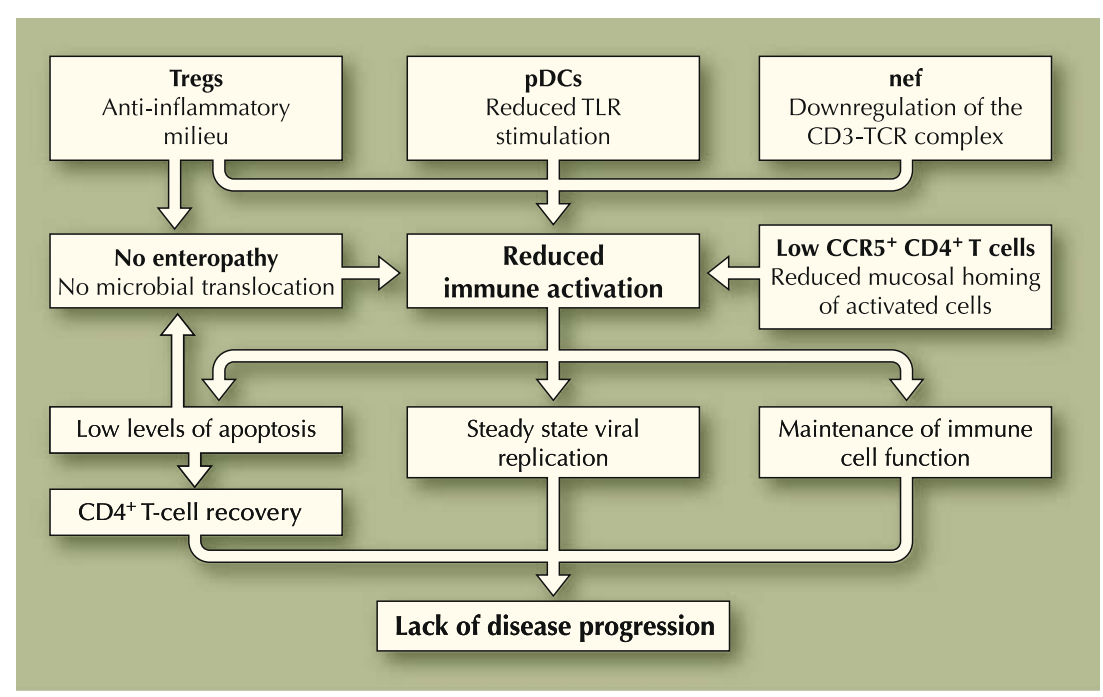


Multiple mechanisms may be responsible for the lack of immune activation in the natural host (Fig. 1):

1) Viral factors, with a potential role for the nef gene products of various SIVs (but not of HIV or SIVcpz) in the protection of natural hosts against chronic immune activation, $\mathrm{CD}^{+}{ }^{+} \mathrm{T}$-cell depletion, and disease progression during natural SIV infection by downregulating the expression of the CD3-TCR complex from the surface of infected $\mathrm{CD}^{+}{ }^{+} \mathrm{T}$ cells [50].

2) The low CCR5 expression on the $\mathrm{CD} 4^{+} \mathrm{T}$ cells may contribute to reduced immune activation and inflammation in the gut, by decreasing the homing of effector memory cells to the intestine.

3) The anti-inflammatory milieu and reduced levels of apoptosis of both lymphocytes and epithelial cells may contribute to the maintenance of a healthy intestinal barrier in natural hosts, thus preventing the release of microbial TLR ligands in circulation [29]. In both AGMs and SMs there is no increase in microbial translocation during SIV infection [29, 51], in great contrast with progressive hosts, such as humans and RMs, in which continuous leakage of microbial components through a severely damaged intestinal mucosa was reported to be responsible for maintaining high levels of immune activation during lentiviral infection [51].

4) Preservation of Th-17 cells in natural hosts during SIV infection may also play an important role in the maintenance of the mucosal barrier, by preserving the epithelial integrity providing protection against intestinal flora $[13,31]$.

5) Preservation of T-regulatory cells in natural hosts may reduce the levels of immune activation either by production of anti-inflammatory cytokines, such as IL-10, or by keeping T-cell proliferation at bay after the initial $\mathrm{CD}^{+} \mathrm{T}$-cell loss. In pathogenic SIV/HIV infections, increased turnover of $\mathrm{T}$ cells together with increased cell death drive exhaustion of T-cell reserves and progression to AIDS.

6) Finally, the innate immune system may also contribute to the low levels of T-cell activation in the natural host, by producing fewer proinflammatory cytokines, by lower activation of the effector function of the adaptive immune system, or by inducing Treg rather than Th1 differentiation.

\section{Conclusions}

African NHP species that are natural hosts of SIVs do not progress to AIDS. Although SIV infection in natural hosts shares numerous features with pathogenic infection, the major difference relies in the effective control of immune activation by natural hosts. Numerous mechanisms have been proposed to be behind this fundamental difference between natural and pathogenic SIV/HIV infections. Intensive research is currently underway to establish which of the pathways leading to the control of immune activation in natural hosts could be exploited in order to control disease progression in HIV-infected patients. It is highly likely that several of these pathways are involved in controlling the deleterious consequences of SIV infection in natural hosts. Also, as these species learned how to cope with SIV infection through a long-term coadaptation, it is equally possible that different species followed different pathways. To date, studies in natural hosts only focused on three animal models, and numerous others are still unexplored. Therefore, all these mechanisms should be investigated in order to identify multiple strategies to keep HIV at bay and control progression to AIDS. An approach that is too simplistic will probably fail, as 20 years of study of natural SIV infection has taught us that "for every complex problem, there is an answer that is clear, simple-and wrong" (H. L. Mencken) [52].

Acknowledgments We thank Françoise Barre-Sinoussi, Jodi Craigo, Beatrice H. Hahn, Vanessa M. Hirsch, Amitinder Kaur, Daniel Douek, Guido Silvestri, Andrew A. Lackner, Alan Landay, Preston A. Marx, Louis Picker, Sentob Saragosti and François Simon for helpful discussions of data and hypotheses. This work was supported by NIH/NIAID/NCRR Grants AI064066 and AI069935 (I.P.), AI065325 (C.A.), and RR025781 (CA/IP). The funders had no role in study design, data collection and analysis, decision to publish, or preparation of the manuscript. We would like to dedicate this manuscript to the memory of Dr. Jonathan S. Allan.

Disclosure No potential conflict of interest relevant to this article was reported.

Open Access This article is distributed under the terms of the Creative Commons Attribution Noncommercial License which permits any noncommercial use, distribution, and reproduction in any medium, provided the original author(s) and source are credited.

\section{References}

Papers of particular interest, published recently, have been highlighted as:

- Of importance

1. Sodora DL, Silvestri G: Immune activation and AIDS pathogenesis. AIDS 2008, 22:439-446.

2. Pandrea I, Sodora DL, Silvestri G, Apetrei C: Into the wild: simian immunodeficiency virus (SIV) infection in natural hosts. Trends Immunol 2008, 29:419-428.

3. VandeWoude S, Apetrei C: Going wild: lessons from Tlymphotropic naturally occurring lentiviruses. Clin Microbiol Rev 2006, 19:728-762. 
4. Apetrei C, Kaur A, Lerche NW, et al.: Molecular epidemiology of SIVsm in US primate centers unravels the origin of SIVmac and SIVstm. J Virol 2005, 79:8991-9005.

5. Pandrea I, Silvestri G, Apetrei C: AIDS in African nonhuman primate hosts of SIVs: a new paradigm of SIV infection. Curr HIV Res 2009, 6:57-72.

6. Pandrea I, Apetrei C, Gordon S, et al.: Paucity of CD4+CCR5+ T cells is a typical feature of natural SIV hosts. Blood 2007, 109:1069-1076.

7. • Pandrea I, Onanga R, Souquiere S, et al.: Paucity of CD4+CCR5+ T-cells may prevent breastfeeding transmission of SIV in natural non-human primate hosts J Virol 2008, 82:5501-5509. This study demonstrated that low CCR5 expression by CD4+ T cells may reduce the levels of SIV mucosal transmission (ie, breastfeeding transmission) in natural hosts.

8. Chen Z, Kwon D, Jin Z, et al.: Natural infection of a homozygous delta24 CCR5 red-capped mangabey with an R2b-tropic simian immunodeficiency virus. J Exp Med 1998, 188:2057-2065.

9. Samson M, Libert F, Doranz BJ, et al.: Resistance to HIV-1 infection in Caucasian individuals bearing mutant alleles of the CCR-5 chemokine receptor gene. Nature 1996, 382:722-725.

10. Gautam R, Gaufin T, Butler I, et al.: SIVrcm, a unique CCR2tropic virus, selectively depletes memory $\mathrm{CD} 4+\mathrm{T}$ cells in pigtailed macaques through rapid coreceptor expansion in vivo. J Virol 2009, 83:7894-7908.

11. - Keele BF, Holland Jones J, Terio K, et al.: Increased mortality and AIDS-like immunopathology in wild chimpanzees infected with SIVcpz. Nature 2009, 460:515-519. This study reported that chimpanzees, which acquired their species-specific SIVs through cross-species transmission (as humans acquired HIV), develop AIDS after SIV infection.

12. Pandrea I, Apetrei C, Dufour J, et al.: Simian immunodeficiency virus (SIV) SIVagm.sab infection of Caribbean African green monkeys: new model of the study of SIV pathogenesis in natural hosts. J Virol 2006, 80:4858-4867.

13. Favre D, Lederer S, Kanwar B, et al.: Critical loss of the balance between Th17 and T regulatory cell populations in pathogenic SIV infection. Plos Pathogens 2009, 5:e1000295.

14. - Beaumier CM, Harris LD, Goldstein S, et al.: CD4 downregulation by memory $\mathrm{CD} 4+\mathrm{T}$ cells in vivo renders African green monkeys resistant to progressive SIVagm infection. Nat Med 2009, 15:879-885. This study reported that downregulation of CD4 by natural hosts results in the emergence of a T-cell subset that is not infected by SIV but maintains the helper function. This may contribute to the lack of disease progression in natural hosts.

15. Murayama Y, Amano A, Mukai R, et al.: CD4 and CD8 expressions in African green monkey helper $\mathrm{T}$ lymphocytes: implication for resistance to SIV infection. Int Immunol 1997, 9:843-851.

16. Murayama Y, Mukai R, Inoue-Murayama M, Yoshikawa Y: An African green monkey lacking peripheral CD4 lymphocytes that retains helper T cell activity and coexists with SIVagm. Clin Exp Immunol 1999, 117:504-512.

17. Kaiser P, Joos B, Niederost B, et al.: Productive human immunodeficiency virus type 1 infection in peripheral blood predominantly takes place in $\mathrm{CD} 4 / \mathrm{CD} 8$ double-negative $\mathrm{T}$ lymphocytes. J Virol 2007, 81:9693-9706.

18. Mandl JN, Barry AP, Vanderford TH, et al.: Divergent TLR7 and TLR9 signaling and type I interferon production distinguish pathogenic and nonpathogenic AIDS virus infections. Nat Med 2008, 14:1077-1087.

19. Diop OM, Ploquin MJ-Y, Mortara L, et al.: Plasmacytoid dendritic cell dynamics and alpha interferon production during simian immunodeficiency virus infection with a nonpathogenic outcome. J Virol 2008, 82:5145-5152.
20. Pandrea I, Silvestri G, Onanga R, et al.: Simian immunodeficiency viruses replication dynamics in African non-human primate hosts: common patterns and species-specific differences. J Med Primatol 2006, 35:194-201.

21. Hirsch VM, Fuerst TR, Sutter G, et al.: Patterns of viral replication correlate with outcome in simian immunodeficiency virus (SIV)-infected macaques: effect of prior immunization with a trivalent SIV vaccine in modified vaccinia virus Ankara. J Virol 1996, 70:3741-3752.

22. Mellors JW, Rinaldo CR Jr, Gupta P, et al.: Prognosis in HIV-1 infection predicted by the quantity of virus in plasma. Science 1996, 272:1167-1170.

23. Apetrei C, Gautam R, Sumpter B, et al.: Virus-subtype specific features of natural SIVsmm infection in sooty mangabeys. J Virol 2007, 81:7913-7923.

24. Pandrea I, Onanga R, Rouquet P, et al.: Chronic SIV infection ultimately causes immunodeficiency in African non-human primates. AIDS 2001, 15:2461-2462.

25. Milush JM, Reeves JD, Gordon S, et al.: Virally-induced CD4+ T cell depletion is not sufficient to induce AIDS in a natural host. J Immunol 2007, 179:3047-3056.

26. - Pandrea I, Gaufin T, Brenchley JM, et al.: Experimentallyinduced immune activation in natural hosts of SIV induces significant increases in viral replication and CD4+ T cell depletion. J Immunol 2008, 181:6687-6691. This provides proof of concept that microbial translocation (modeled here by lipopolysaccharide administration) results in increased immune activation, increased viral replication, and CD4+T-cell depletion.

27. Klatt NR, Villinger F, Bostik P, et al.: Availability of activated $\mathrm{CD} 4+\mathrm{T}$ cells dictates the level of viremia in naturally SIVinfected sooty mangabeys. J Clin Invest 2008, 118:2039-2049.

28. Broussard SR, Staprans SI, White R, et al.: Simian immunodeficiency virus replicates to high levels in naturally infected African green monkeys without inducing immunologic or neurologic disease. J Virol 2001, 75:2262-2275.

29. Pandrea I, Gautam R, Ribeiro R, et al.: Acute loss of intestinal CD4+ T cells is not predictive of SIV virulence. J Immunol 2007, 179:3035-3046.

30. Gordon S, Klatt NR, Milush JM, et al.: Severe depletion of mucosal CD4+ $\mathrm{T}$ cells in AIDS-free SIV-infected sooty mangabeys. J Immunol 2007, 179:3026-3034.

31. Brenchley JM, Paiardini M, Knox KS, et al.: Differential Th17 CD4 T-cell depletion in pathogenic and nonpathogenic lentiviral infections. Blood 2008, 112:2826-2835.

32. Pandrea I, Ribeiro RM, Gautam R, et al.: Simian immunodeficiency virus SIVagm dynamics in African green monkeys. J Virol 2008, 82:3713-3724.

33. Gordon S, Dunham RM, Engram JC, et al.: Short-lived infected cells support the bulk of virus replication in naturally SIV-infected sooty mangabeys: implications for AIDS pathogenesis. J Virol 2008, 82:3725-3735.

34. Pandrea I, Kornfeld C, Ploquin MJ-I, et al.: Impact of viral factors on very early in vivo replication profiles in SIVagm-infected African green monkeys. J Virol 2005, 79:6249-6259.

35. Saez-Cirion A, Pancino G, Sinet M, et al.: HIV controllers: how do they tame the virus? Trends Immunol 2007, 28:532-540.

36. Hirsch VM: What can natural infection of African monkeys with simian immunodeficiency virus tell us about the pathogenesis of AIDS? AIDS Rev 2004, 6:40-53.

37. Lozano Reina JM, Favre D, Kasakow Z, et al.: Gag p27-specific Band T-cell responses in simian immunodeficiency virus SIVagminfected African green monkeys. J Virol 2009, 83:2770-2777.

38. Gicheru MM, Otsyula M, Spearman P, et al.: Neutralizing antibody responses in African green monkeys naturally infected with simian immunodeficiency virus (SIVagm). J Med Primatol 1999, 28:97-104. 
39. Gaufin T, Pattison M, Gautam R, et al.: Effect of B cell depletion on viral replication and clinical outcome of SIV infection in a natural host. J Virol 2009, 83:10347-10357.

40. Gaufin T, Gautam R, Kasheta M, et al.: Limited ability of humoral immune responses in control of viremia during infection with SIVsmmD215 strain. Blood 2009, 113:4250-4261.

41. Kaur A, Alexander L, Staprans SI, et al.: Emergence of cytotoxic $\mathrm{T}$ lymphocyte escape mutations in nonpathogenic simian immunodeficiency virus infection. Eur J Immunol 2001, 31:3207-3217.

42. Zahn RC, Rett MD, Korioth-Schmitz B, et al.: Simian immunodeficiency virus (SIV)-specific CD8 $+\mathrm{T}$ cell responses in chronically SIVagm-infected vervet African green monkeys. J Virol 2008, 82:11577-11588.

43. Wang Z, Metcalf B, Ribeiro RM, et al.: Th-1-type cytotoxic CD8+ T-lymphocyte responses to simian immunodeficiency virus (SIV) are a consistent feature of natural SIV infection in sooty mangabeys. J Virol 2006, 80:2771-2783.

44. Schmitz JE, Zahn RC, Brown CR, et al.: Inhibition of adaptive immune responses leads to a fatal clinical outcome in SIV-infected pigtailed macaques but not vervet African green monkeys. PLoS Pathog 2009, In revision.

45. Barry AP, Silvestri G, Safrit JT, et al.: Depletion of CD8+ cells in sooty mangabey monkeys naturally infected with simian immunodeficiency virus reveals limited role for immune control of virus replication in a natural host species. J Immunol 2007, 178:80028012.

46. Kaur A, Di Mascio M, Barabasz A, et al.: Dynamics of T- and Blymphocyte turnover in a natural host of simian immunodeficiency virus. J Virol 2008, 82:1084-1093.

47. Meythaler M, Martinot A, Wang Z, et al.: Differential CD4+ Tlymphocyte apoptosis and bystander T-cell activation in rhesus macaques and sooty mangabeys during acute simian immunodeficiency virus infection. J Virol 2009, 83:572-583.

48. Bostik P, Dodd GL, Ansari AA: CD4 + T cell signaling in the natural SIV host-implications for disease pathogenesis. Front Biosci 2003, 8:s904-s912.

49. Kornfeld C, Ploquin MJ, Pandrea I, et al.: Antiinflammatory profiles during primary SIV infection in African green monkeys are associated with protection against AIDS. J Clin Invest 2005, 115:1082-1091.

50. Schindler M, Munch J, Kutsch O, et al.: Nef-mediated suppression of $\mathrm{T}$ cell activation was lost in a lentiviral lineage that gave rise to HIV-1. Cell 2006, 125:1055-1067.

51. Brenchley JM, Price DA, Schacker TW, et al.: Microbial translocation is a cause of systemic immune activation in chronic HIV infection. Nat Med 2006, 12:1365-1371.

52. Mencken HL: The Divine Afflatus. New York Evening Mail. November 16, 1917. 